\title{
Diagnostic Performance of Soluble Triggering Receptor Expressed on Myeloid Cells-1 in Ventilator-Associated Pneumonia of Patients with Ischemic Stroke
}

\author{
Yuetian Yu, ${ }^{1}$ Cheng Zhu, ${ }^{2}$ Chunyan Liu, ${ }^{3}$ Yuan Gao, ${ }^{1}$ Rong Yin, ${ }^{1}$ and Jianguo Cao ${ }^{1}$ \\ ${ }^{1}$ Department of Critical Care Medicine, Renji Hospital, School of Medicine, Shanghai Jiao Tong University, Shanghai 200001, China \\ ${ }^{2}$ Department of Emergency Medicine, Ruijin Hospital, School of Medicine, Shanghai Jiao Tong University, Shanghai 200025, China \\ ${ }^{3}$ Department of Emergency Medicine, Minhang Central Hospital, School of Medicine, Fudan University, Shanghai 201100, China
}

Correspondence should be addressed to Rong Yin; heroyin2000@outlook.com and Jianguo Cao; doctorcjg@sina.com

Received 14 September 2016; Revised 26 November 2016; Accepted 20 December 2016; Published 22 February 2017

Academic Editor: Giordano Dicuonzo

Copyright (C) 2017 Yuetian Yu et al. This is an open access article distributed under the Creative Commons Attribution License, which permits unrestricted use, distribution, and reproduction in any medium, provided the original work is properly cited.

\begin{abstract}
Objective. To investigate the effect of soluble triggering receptor expressed on myeloid cells-1 (sTREM-1) in serum, bronchoalveolar lavage fluid (BALF), endotracheal aspiration (ETA), and exhaled breath condensate (EBC) samples as early biomarkers for the diagnosis of ventilator-associated pneumonia (VAP) in patients with ischemic stroke. Methods. One hundred and thirty-two patients with clinically suspected VAP were enrolled in this study. Bronchoscopy was performed on the day of clinically suspected VAP. sTREM-1 levels in serum, BALF, ETA, and EBC were measured. VAP was diagnosed by quantitative cultures of BALF $\left(\geq 10^{4} \mathrm{cfu} / \mathrm{mL}\right)$. Results. VAP was confirmed in 76 (57.58\%) cases. Patients with VAP showed significantly higher sTREM-1 in BALF [32.35 (IQR, 30.08-41.72) versus $18.92(11.89-31.72)] \mathrm{pg} / \mathrm{mL}$ and in EBC [1.57 (IQR, 1.02-2.61) versus 0.41 (0.19-1.61)] pg/mL than patients without VAP. The area under the curve was $0.813(p<0.001)$. The optimum cut-off value for sTREM-1 in BALF was $23.61 \mathrm{pg} / \mathrm{mL}$, yielding sensitivity and specificity of $85.5 \%$ and $73.1 \%$. sTREM-1 in BALF had excellent correlation with that in EBC $\left(R^{2}=0.78, p<0.05\right)$. Conclusions. sTREM-1 in EBC and BALF had good diagnostic performance in differentiating patients with and without VAP.
\end{abstract}

\section{Introduction}

Ventilator-associated pneumonia (VAP) which constitutes a frequent infection in intensive care unit (ICU) patients consumes vast healthcare resources and increases proportionally to the duration of ICU stay and mortality [1]. The incidence of VAP varies depending on the study population. For example, in patients with ischemic stroke who are characterized by advanced age, depressed level of consciousness, immune suppression, and long-term bed rest, the incidence of VAP can increase to approximately $40 \%$, which is associated with a less favorable neurologic and functional outcome [2]. It is recognized that one-third to half of all VAP-related deaths are directly attributable to pneumonia. Diagnosis of VAP with clinical suspicion is overly sensitive with low specificity, leading to unnecessary antibiotics use [3], and the incidence of VAP among ICU ischemic stroke patients has not been thoroughly investigated.
The triggering receptor expressed on myeloid cells-1 (TREM-1) is a member of the immunoglobulin superfamily. Its expression on phagocytes is upregulated by exposure to bacteria and fungi. A soluble form of TREM-1 (sTREM-1) was proposed as a new biomarker which had been tested for acute infections with different diagnostic and prognostic value [4]. sTREM- 1 can be found in different body fluids, such as serum, bronchoalveolar lavage fluid (BALF), endotracheal aspiration (ETA), and exhaled breath condensate (EBC), where it can be assayed by ELISA using commercial immunoassay kits [5]. Some clinical studies have proved that sTREM-1 did have the ability to identify patients with sepsis while others come to an opposite conclusion $[6,7]$.

However, the real effect of sTREM-1 on diagnosis of VAP is still unknown and has not been well evaluated yet. Furthermore, the majority of the studies about sTREM-1 levels in VAP were heterogeneous due to including different diseases. What is the most important is that some of these studies 
did not exclude the patients with extrapulmonary infection which might lead to misinterpretation of the study results. The subjects of our study were patients with ischemic stroke, who had a high incidence of VAP and were without any infectious complications at the time of invasive mechanical ventilation.

Trauma, malignant neoplasms, and acute inflammatory response could all lead to the raise of sTREM-1 in serum due to its high sensitivity and low specificity which resulted in the poor performance in VAP diagnosis. BALF was partly secreted by lung which was infected as the target organ. So we measured sTREM-1 levels in serum, BALF, ETA, and EBC samples from patients who underwent bronchoscopy for a clinical suspicion of VAP and we hypothesized that sTREM-1 in different body fluid might have different values in diagnosing VAP.

\section{Methods}

2.1. Research Briefs. The present study was a multicenter (155 ICU beds in total) prospective observational trial which included samples of serum, BALF, ETA, and EBC from eligible patients. The study was approved by the Review Board and Ethics Committee of Shanghai Jiaotong University School of Medicine (No: 2013-Clinical-Res-085) and informed consent was obtained for all patients, from either the patient or the next of kin. The study was performed from January 2013 to December 2015. One month before the start of this study, a standardized sampling, processing, analysis, and statistics procedure was set by 9 investigators from these centers after 3 days' learning and discussion. All of the centers carefully followed this procedure during the study time. There was no significant difference in compliance among the centers.

2.2. Study Population. Consecutive sampling was used to recruit critical ill patients. Patients were eligible for enrolment if they were diagnosed with ischemic stroke by magnetic resonance imaging (MRI), underwent bronchoscopy while mechanically ventilated for clinically suspected VAP, and aged between 18 and 80 years. Patients were excluded from the study if they were moribund or not expected to survive 3 days because of an underlying irreversible medical condi tion, had active pneumonia when admitted to ICU or had extrapulmonary infection during ICU stay, were immunocompromised, were with malignancies, and were pregnant or if informed consent could not be obtained.

2.3. Diagnosis, Treatment, and Prevention of VAP. VAP was suspected if the patient had a radiographic infiltrate that was new or progressive, together with clinical findings that were suggestive of infection, such as the onset of fever (temperature $\left.\geq 38.3^{\circ} \mathrm{C}\right)$, leukocytosis $\left(\geq 10 \times 10^{9} / \mathrm{L}\right.$ or $\left.\leq 4 \times 10^{9} / \mathrm{L}\right)$, purulent sputum, and decline in oxygenation. In addition, a positive BAL culture ( $\geq 10^{4}$ colony-forming units $/ \mathrm{mL}$, cfu/mL) was required to confirm the diagnosis of VAP [8]. The protocol for VAP treatment and prevention followed standard protocols in all institutions also based on accepted guidelines. Care bundles for preventing VAP were also employed $[3,8]$.
2.4. Clinical Assessment. Baseline assessment included the evaluation of demographic data (age and gender), medical history, stroke subtype, ratio of partial oxygen to fraction of inspired oxygen $\left(\mathrm{PaO}_{2} / \mathrm{FiO}_{2}\right)$, acute physiology and chronic health evaluation (APACHE II), Glasgow Coma Scale (GCS), modified clinical pulmonary infection score (mCPIS), and the level of inflammatory biomarkers.

2.5. Samples Processing and Measurement. Bronchoscopy was performed on the day when VAP was suspected and all samples were collected for measurement at the same day. The diagnostic flexible bronchoscopy guideline of British Thoracic Society was also followed during the performance [9]. Selection of the segment for bronchoalveolar lavage (BAL) was guided by chest X-ray changes. The right middle lobe or lingual lobe was selected when diffuse infiltrates were present. Five aliquots of $20 \mathrm{~mL}$ of sterile saline were instilled and aspirated gently. The first aliquot was discarded and the subsequent four aliquots were pooled for analysis. Part of the first BALF specimen was sent to the laboratory immediately after collection for measurement of sTREM-1 levels; the remainder of the sample was sent to the microbiology laboratory for quantitative culture. Quantitative culture of BALF retrieved by direct bronchoscopic methods yields the best sensitivity and specificity to diagnose VAP and can differentiate true infection from colonization or inflammation. Culture-positive BALF was defined as a count $\geq 10^{4} \mathrm{cfu} / \mathrm{mL}$. Thus, the diagnosis of pneumonia was confirmed by a positive BALF culture [10].

The EBC fluid was collected in a plastic container located in the center of the exhaled portion of the ventilator tubing (mid-way between the ventilator and the patient's endotracheal tube). EBC was centrifuged and cell-free supernatants were aliquoted into 2 polypropylene tubes and stored at $-80^{\circ} \mathrm{C}$.

ETA fluid was collected using a feeding tube, which was introduced down the endotracheal tube until resistance was felt. Then, using a syringe, $1 \mathrm{ml} / \mathrm{kg}$ of normal saline (maximum $20 \mathrm{ml}$ ) was instilled through the feeding tube followed by a small amount of air to clear the dead space of the endotracheal tube and suction was applied to obtain the fluid [11].

Blood samples $(5 \mathrm{~mL})$ from median basilic vein were separated by centrifugation (TDL-60C, 6000 revolutions per minute, China) and stored at $-80^{\circ} \mathrm{C}$ in anticoagulative tubes.

Commercially available ELISA assays were used for detecting sTREM-1. The detection limit of the methods for sTREM-1 was $0.05 \sim 1600 \mathrm{pg} / \mathrm{mL}$ (SEA571Hu96 Tests, USA). All samples were run in duplicate. To minimize variations in samples collection, the procedure was performed by the same investigator.

2.6. Statistical Analysis. Statistical analysis was performed using SPSS version 19.0 (IBM for Windows). Data were initially assessed for normality and log transformed where appropriate. Data between the VAP positive and VAP negative were compared using Chi-square test for equal proportion or Fisher exact test where numbers were small with 
TABLE 1: Characteristics of the study groups (mean $\pm \mathrm{SD} / \% / \mathrm{IQR})$.

\begin{tabular}{|c|c|c|c|c|}
\hline Characteristics & All patients $(n=132)$ & Patients with VAP $(n=76)$ & Patients without VAP $(n=56)$ & $p$ \\
\hline Age, yrs & $70.27 \pm 11.43$ & $69.39 \pm 10.84$ & $70.89 \pm 11.36$ & 0.447 \\
\hline Male gender, $n(\%)$ & $63(47.7)$ & $37(48.7)$ & $26(46.4)$ & 0.861 \\
\hline \multicolumn{5}{|l|}{ Previous medical illness } \\
\hline Cardiovascular disease, $n(\%)$ & $70(53.03)$ & $39(51.31)$ & $31(55.35)$ & 0.725 \\
\hline Cerebrovascular disease, $n(\%)$ & $41(31.06)$ & $23(30.26)$ & $18(32.14)$ & 0.851 \\
\hline \multicolumn{5}{|l|}{ Position of stroke } \\
\hline TACI, $n(\%)$ & $48(36.36)$ & $29(38.16)$ & $19(33.93)$ & 0.715 \\
\hline POCI, $n(\%)$ & $84(63.64)$ & $47(61.84)$ & $37(66.07)$ & 0.715 \\
\hline Smoking history & $39(29.55)$ & $24(31.58)$ & $15(26.78)$ & 0.571 \\
\hline Hypoproteinemia & $32(24.24)$ & $17(22.37)$ & $15(26.79)$ & 0.682 \\
\hline APACHE II & $17.92 \pm 4.94$ & $18.21 \pm 5.48$ & $17.52 \pm 4.88$ & 0.442 \\
\hline GCS & $5.71 \pm 2.27$ & $5.82 \pm 2.35$ & $5.69 \pm 2.78$ & 0.085 \\
\hline mCPIS & $3.42 \pm 1.45$ & $3.37 \pm 1.28$ & $3.45 \pm 1.61$ & 0.751 \\
\hline $\mathrm{PO}_{2} / \mathrm{FiO}_{2}(\mathrm{mmHg})$ & $112.67 \pm 50.11$ & $115.75 \pm 48.58$ & $108.92 \pm 52.78$ & 0.443 \\
\hline
\end{tabular}

TACI, total anterior circulation infarcts; POCI, posterior circulation infarcts; APACHE II, acute physiology and chronic health evaluation; GCS, Glasgow Coma Scale; mCPIS, modified clinical pulmonary infection score.

results presented as percentages $(n)$. Continuously normally distributed variables were compared using Student's $t$-test and presented as means (standard deviations), whereas nonnormally distributed data was compared using Wilcoxon rank-sum test and reported as medians (interquartile range). The statistical tests performed were two-sided. All analysis was performed on an intention-to-treat basis and a twosided $p<0.05$ was considered to be statistically significant. Figures were drawn using Graphpad prism version 5.0 and Medcalc11.4.2.

\section{Results}

3.1. Characteristics of the Patients. Over the study period, 328 patients with acute ischemic stroke and requiring mechanical ventilation were admitted to ICU. Thirty-two patients were excluded because informed consent was not obtained. One hundred and sixty-four patients who had active pneumonia when admitted or had extrapulmonary infection during ICU stay were also excluded. One hundred and thirty-two patients clinically suspected of VAP were included in the study. Among them 76 (57.58\%) were diagnosed of VAP while 56 were not. The baseline characteristics of the 132 patients were shown in Table 1. The demographic data, position of stroke, $\mathrm{PaO}_{2} / \mathrm{FiO}_{2}$, APACHE II, GCS, and modified CPIS were not significantly different between patients with or without VAP $(p>0.05)$.

3.2. Bacteria Detection. BALF culture was positive (considering the cut-off of $>10^{4} \mathrm{cfu} / \mathrm{mL}$ ) in all the 76 patients with VAP, with the growth of the following agents: Gram-negative bacterium ( $n=62,81.58 \%$ ). The top three Gram-negative bacteria were Pseudomonas aeruginosa $(n=28)$, Klebsiella pneumoniae $(n=15)$, and Acinetobacter baumannii $(n=$ 11). Five Methicillin-resistant Staphylococcus aureus were also detected in 14 strains of Gram-positive bacterium. Blood culture was positive in 4 patients with VAP, with the growth of Staphylococcus aureus methicillin-resistant $(n=3)$ and Staphylococcus aureus methicillin-sensitive $(n=1)$.

3.3. sTREM-1 Detection and Comparison. sTREM-1 was detected in all the 132 patients at the day of VAP suspicion. The levels of sTREM-1 in serum, BALF, ETA, and EBC between two groups were compared. BALF sTREM-1 concentrations were higher in patients with VAP than patients without VAP [32.35 (IQR, 30.08-41.72) versus 18.92 (11.89$31.72)] \mathrm{pg} / \mathrm{mL}$ as well as EBC sTREM-1 concentrations $[1.57$ (IQR, 1.02-2.61) versus $0.41(0.19-1.61)] \mathrm{pg} / \mathrm{mL}(p<0.05)$. sTREM-1 levels were consistently higher in BALF than EBC in all patients.

Differences of sTREM-1 levels in serum and ETA between two groups were not statistically significant $(p>0.05)$ (Figure 1).

There were 56 patients who were not VAP among the 132 patients included in our study. In these 56 patients, the average time of suspected VAP was about $76 \mathrm{~h}$. We then also included another 56 patients who needed invasive mechanical ventilation during our study period to be a negative control group. None of the 56 patients in the control group was with infectious diseases. We compared the sTREM-1 levels in $76 \mathrm{~h}$ of our 56 non-VAP patients with the negative control group and found that the sTREM-1 concentrations in serum [322.94 (IQR, 276.62-427.72) versus 308.69 (284.77418.93)] pg/mL, BALF [18.92 (IQR, 11.89-31.72) versus 17.98 (10.92-33.60)] pg/mL, ETA [680.53 (IQR, 642.87-718.72) versus $673.98(657.82-715.21)] \mathrm{pg} / \mathrm{mL}$, and EBC [0.41 (IQR, 0.191.61) versus $0.46(0.17-1.73)] \mathrm{pg} / \mathrm{mL}$ were similar. The results proved that the 56 patients in our study did not have any infectious disease during our study period indeed.

3.4. sTREM-1 Sensitivity and Specificity and Diagnostic Values for VAP. Receiver-operating characteristic curve (ROC) was 
TABLE 2: Best cut-off values of sTREM-1 that were obtained from ROC curves for VAP diagnosis.

\begin{tabular}{lccccc}
\hline Variables & AUC & $95 \%$ CI & Cut-off $(\mathrm{pg} / \mathrm{mL})$ & Sensitivity\% & Specificity\% \\
\hline sTREM-1 in serum & 0.528 & $0.438 \sim 0.617$ & 358.93 & 64.4 & 85.9 \\
sTREM-1 in ETA & 0.551 & $0.461 \sim 0.638$ & 23.61 & 85.5 & 28.8 \\
sTREM-1 in BALF & 0.831 & $0.755 \sim 0.892$ & 0.32 & 100 & 73.1 \\
sTREM-1 in EBC & 0.752 & $0.668 \sim 0.824$ & 48.1 \\
\hline
\end{tabular}

$\mathrm{AUC}=$ area under the curve.
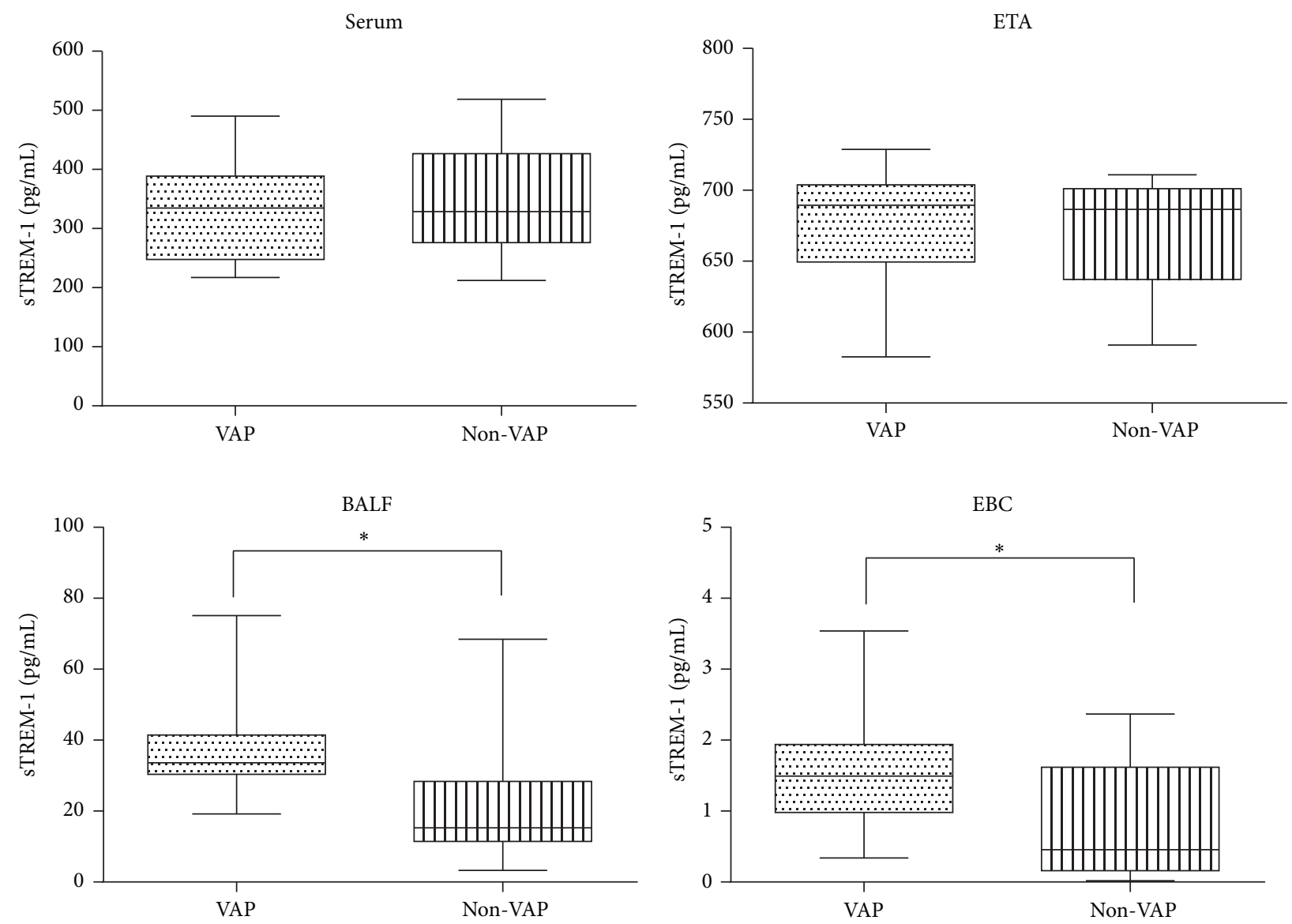

FIGURE 1: Median soluble triggering receptor expressed on myeloid cells-1 (sTREM-1) levels in serum, endotracheal aspiration (ETA), bronchoalveolar lavage fluid (BALF), and exhaled breath condensate (EBC) samples in 76 patients with and 56 patients without ventilatorassociated pneumonia (VAP). In the data bars, the mid-lines represent medians; the tops and bottoms of the bars represent the 25th and 75th percentiles; ${ }^{*} p<0.05$.

shown in Figure 2. A cut-off value of $23.61 \mathrm{pg} / \mathrm{mL}$ for sTREM1 in BALF resulted in a sensitivity of $85.5 \%$ and specificity of $73.1 \%$ which corresponded to the area of 0.831 under the ROC. A cut-off value of $0.31 \mathrm{pg} / \mathrm{mL}$ for sTREM-1 in EBC resulted in a relative high sensitivity of $98.3 \%$ and low specificity of $48.1 \%$ which corresponded to the area of 0.752 under the ROC. The area under the ROC of serum sTREM-1 concentrations was 0.528 and 0.551 of ETA, which were both having low sensitivity and specificity (Table 2 ).

3.5. Correlation of sTREM-1 in Different Samples. A scatter plot was employed to determine whether there were correlations of sTREM-1 between different samples. The results showed that there was a significant correlation between BALF and EBC sTREM-1 levels $\left(R^{2}=0.78, Y=-0.16+0.046 X, p<\right.$ $0.05)$. No statistically significant correlation was identified between the ranks of BALF and serum sTREM-1 values $(Y=$ $300.81+1.483 X)$ or the ranks of BALF and ETA sTREM-1 values $(Y=647.01+0.023 X), p>0.05$, Figure 3 .

\section{Discussion}

4.1. Key Findings. In this prospective multicenter study, all the 132 patients with acute ischemic stroke and suspected VAP underwent bronchoscopy detection. Samples of serum, BALF, ETA, and EBC of these patients were collected and 


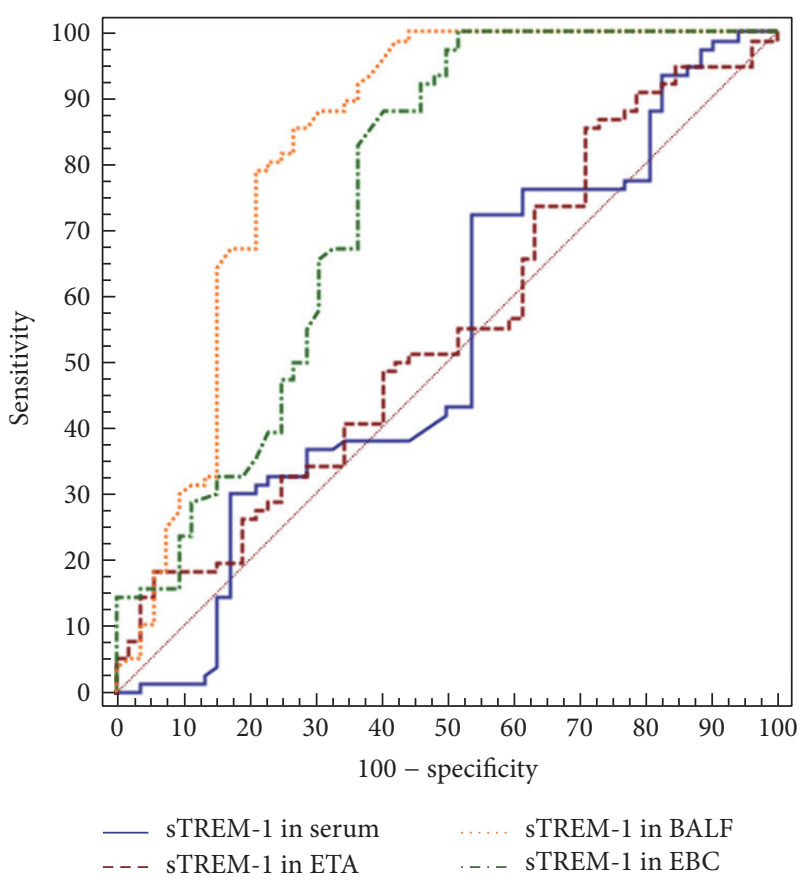

FIGURE 2: Receiver-operating characteristic curves (ROC) of soluble triggering receptor expressed on myeloid cells-1 (sTREM-1) in serum, endotracheal aspiration (ETA), bronchoalveolar lavage fluid (BALF), and exhaled breath condensate (EBC) samples.

analyzed. We found that the concentration of sTREM-1 in BALF and EBC could effectively categorize patients as VAP positive or VAP negative when using direct bronchoscopic quantitative culture samples as the comparison standard and they had good correlation $\left(R^{2}=0.78\right)$. To the best of our knowledge, this is the first study which simultaneously detected concentration of sTREM-1 in serum, ETA, BALF, and $\mathrm{EBC}$ in patients who were suspected of VAP.

4.2. Relationship to Previous Studies. Despite the major advances that have been achieved in prevention and diagnosis of VAP in the last few years, there are still a large number of unanswered questions. The true incidences of VAP in high risk patients, especially those with ischemic stroke in the ICU, are yet to be determined.

What is more, the diagnostic value of sTREM-1 as a biomarker in determining VAP was controversial. A recent study confirmed that STREM-1 levels did have diagnostic and prognostic values in VAP [12]. However, other studies reported poor sensitivity and specificity of sTREM-1 to detect patients with pneumonia [13, 14]. An important explanation for the disparity lies in the difference in study populations. Some studies did not exclude patients who were immunocompromised which might lead to false negative results of sTREM-1 assay. Others did not exclude the patients who had extrapulmonary infection during ICU stay which increased the sTREM-1 concentration that was not the result of VAP. The value of BALF sTREM- 1 in pneumonia was also controversial because of the noninfective influence factors, such as tuberculosis and connective tissue diseases [15].
Additionally, an earlier study indicated that it was hard to classify patients as VAP positive or VAP negative by using CPIS and sTREM-1 [16].

Procalcitonin (PCT) is a prohormone secreted into serum most likely from neuroendocrine cells in the lungs or intestine as part of the systemic inflammatory response. The rapid release and long half-life of procalcitonin make it potentially useful as a diagnostic indicator of VAP. Four studies report the use of serum PCT as a biomarker in diagnosing VAP [1720]. These studies revealed that sensitivities ranged between 41 and $100 \%$ with lower sensitivities indicating the potential to miss many positive VAP patients. Specificity was higher in two of four studies with a range of $97-100 \%$. Variable cut-off values and dissimilar study designs across the studies contribute to the difficulty in interpreting the results. The different patient populations across studies may have contributed to elevated PCT levels that were unrelated to VAP. The most important thing is that some interference factors like previous antibiotics use did affect the mean serum PCT level and sensitivity and specificity which suggested that serum PCT was not a good biomarker for VAP [21].

Therefore, new biomarkers are urgently needed to diagnose VAP especially for patients with ischemic stroke due to its high mortality. So we detected the concentrations of sTREM-1 and expected that it had higher sensitivity and specificity than PCT.

4.3. Study Significance. Biomarkers can be detected in any biological sample including serum, ETA, BALF, and EBC. A biomarker for VAP should be low or absent when infection is not present and elevated in the presence of infection. sTREM-1 belongs to the immunoglobulin superfamily, which is expressed in neutrophils, monocytes, and macrophages in the course of acute inflammatory response. sTREM-1 triggers the secretion of proinflammatory mediators through a signaling pathway (DAP12) and functions as an amplifier of the inflammatory response. This characteristic leads to its high sensitivity and poor specificity in infectious diseases. In response to infection, sTREM-1 is either secreted or shed and can be measured in body fluids and is almost undetectable in patients with nonmicrobial inflammation. So in patients with pneumonia, it is a better choice to detect the concentration of sTREM-1 in BALF than in serum which leads to a relative higher specificity.

One previous study reported that sTREM-1 increased in serum samples of patients with pneumonia and in EBC samples of patients with VAP [15]. Moreover, the raise of sTREM-1 level in pleural effusion, cerebrospinal fluid, urine, and synovial fluid also has important clinical significance in identifying the infectious diseases [22]. Therefore, sTREM1 as a biomarker for the diagnosis of inflammatory diseases is of high sensitivity and low specificity. According to the characteristics of sTREM-1 expression, it is concluded that analyses of serum sample alone were not able to distinguish between the systemic acute inflammatory response and local infection accurately, although in some patients it could reflect the severity of the infection especially in neonatal sepsis [23]. In our study, the specificity of serum sTREM-1 in diagnosing 


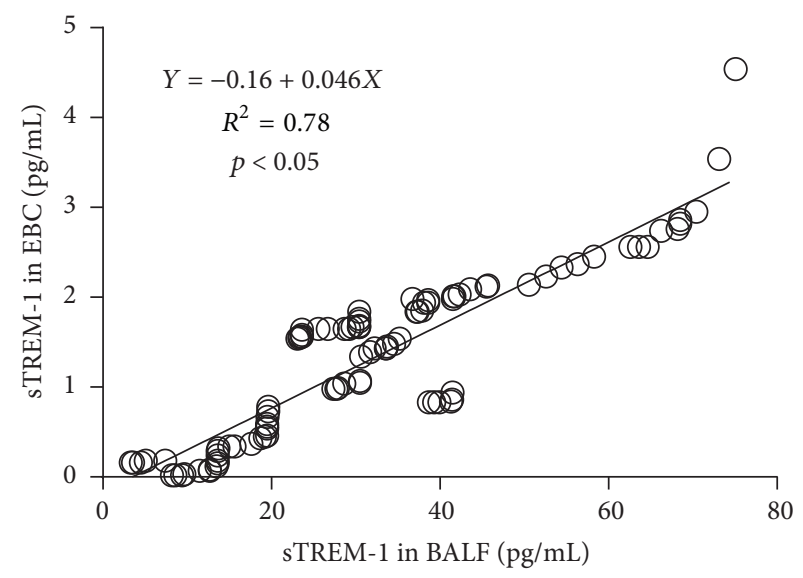

(a)

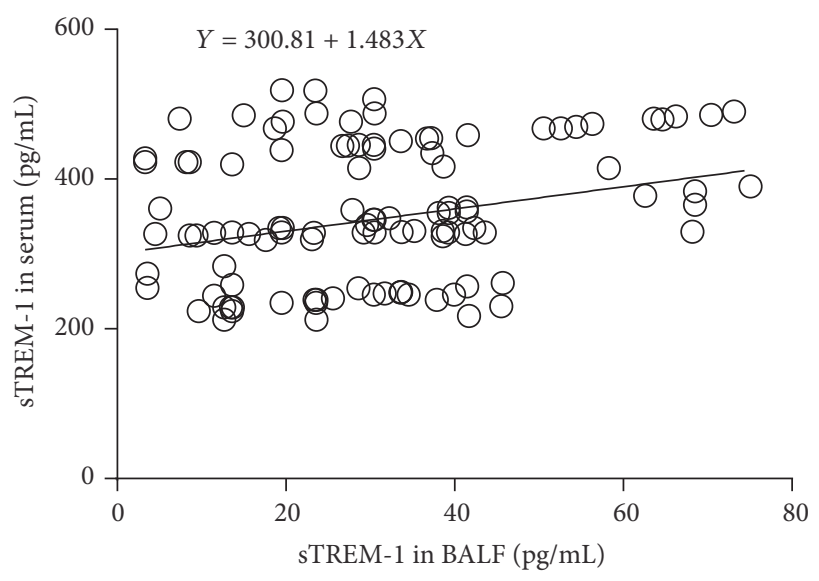

(b)

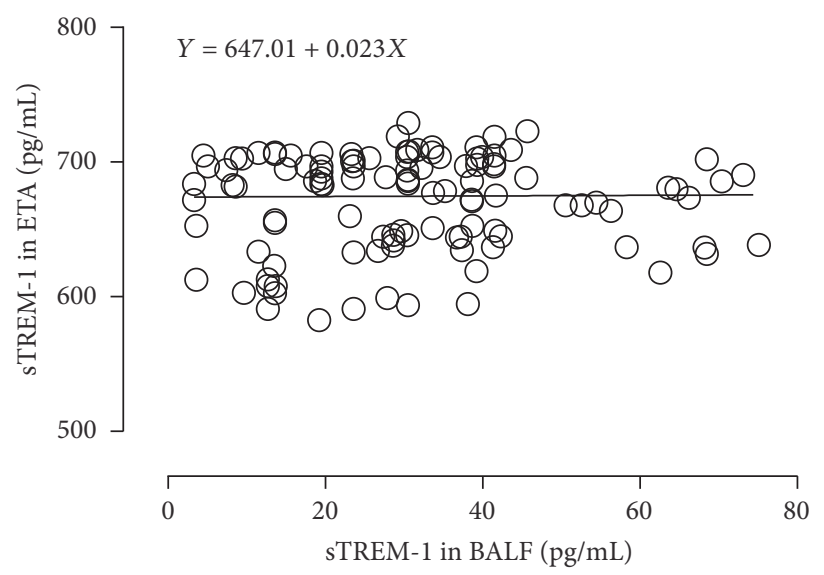

(c)

FIGURE 3: Correlation of sTREM-1 in different samples. (a) Correlation of BALF and EBC sTREM-1 concentration. (b) Correlation of BALF and serum sTREM-1 concentration. (c) Correlation of BALF and ETA sTREM-1 concentration.

VAP was only $46.2 \%$. We acquired ETA samples via the artificial airway (endotracheal tube) which could inevitably be contaminated by the bacterial colonization, leading to misinterpretation of the study results. Therefore, our study also analyzed sTREM-1 levels in BALF and EBC in order to achieve more accurate evaluation.

Damage of the tracheal mucous membrane and stress could all lead to the raise of sTREM-1 in serum due to its high sensitivity and low specificity which resulted in the poor performance in VAP diagnosis. The area under the ROC is only 0.528 . The incidence of VAP will increase along with the incidence of bacterial colonization when mechanical ventilation was prolonged which will lead to the false positive result of sTREM-1 in ETA. In our study, the specificity of sTREM-1 in ETA was only $28.8 \%$, which could not diagnose VAP accurately.

sTREM-1 as an inflammatory biomarker could be detected in epithelial lining fluid which could also be detected in BALF indirectly that was secreted by lung which was infected as the target organ. Detection of sTREM-1 in BALF was a more effective way to effectively categorize patients as VAP positive or VAP negative and it was less intrusive to extrapulmonary infection. Our study also confirmed that on the day of clinically suspected VAP sTREM-1 in BALF had high sensitivity and specificity with 0.831 area under the ROC which indicated that it had higher diagnostic accuracy of VAP. An increasing number of researches on EBC in the diseases of chronic obstructive pulmonary disease, asthma, and other airway inflammatory diseases were published [2426]. However, studies on EBC in pneumonia or VAP were relatively rare. What is more, the results of these studies were still controversial because the methods of EBC acquisition, preservation, and detection have not been standardized.

EBC fluid was collected in a plastic container located at the center of the exhaled portion of the ventilator tubing that was far away from the artificial airway. Although the level of EBC was relatively low because it was composed of expiratory air, it had a high sensitivity (98.3\%) in diagnosing VAP. However, detection and analysis of EBC were inevitably affected by the bacterial colonization in the artificial airway, which resulted in an unsatisfactory specificity (48.1\%). At all events, the area under ROC of sTREM-1 in EBC could reach 0.752 , and the concentration of sTREM-1 in EBC had good correlation with sTREM-1 in BALF $\left(R^{2}=0.78\right)$, 
indicating that it might be one of the accessory biomarkers in diagnosing VAP. As the collection of EBC is noninvasive, it is promising in clinical practice in the future with the standardized methods.

This study has several limitations. We included a homogeneous population of ischemic stroke which might minimize the heterogeneity and avoided misinterpreting the study result. However, its application to routine clinical practice remains uncertain. Whether the detection of BALF sTREM1 in general may translate into clinical benefits outside this setting deserves further explorations.

\section{Conclusion}

In conclusion, sTREM-1 levels in BALF and EBC accurately distinguished patients of acute ischemic stroke with or without VAP when using direct bronchoscopic quantitative culture samples as the reference standard. We can expect the routine use of sTREM-1 levels in BALF or EBC to diagnose patients with VAP in the future with the standardization of samples improvement.

\section{Abbreviations}

APACHE II: Acute physiology and chronic health evaluation

BAL: Bronchoalveolar lavage

BALF: $\quad$ Bronchoalveolar lavage fluid

CFU: Colony-forming units

CPIS: Clinical pulmonary infection score

EBC: $\quad$ Exhaled breath condensate

ELISA: Enzyme-linked immunosorbent assay

ETA: $\quad$ Endotracheal aspiration

GCS: Glasgow Coma Scale

ICU: Intensive care unit

IQR: Interquartile range

PCT: Procalcitonin

POCI: Posterior circulation infarcts

ROC: Receiver-operating characteristic curves

sTREM-1: Soluble triggering receptor expressed on myeloid cells-1

TACI: Total anterior circulation infarcts

VAP: Ventilator acquired pneumonia.

\section{Competing Interests}

The authors have declared that no competing interests exist.

\section{Authors' Contributions}

Yuetian Yu and Cheng Zhu both conceived and designed the experiments. Yuetian Yu, Cheng Zhu, Chunyan Liu, and Rong Yin performed the experiments. Yuetian Yu and Rong Yin analyzed the data. Cheng Zhu and Rong Yin contributed reagents/materials/analysis tools. Yuetian Yu and Cheng Zhu both helped to draft and edit the article. Yuan Gao and
Jianguo Cao revised and approved the final manuscript. Yuetian Yu and Cheng Zhu contributed equally to this work.

\section{Acknowledgments}

The authors appreciate Zhongheng Zhang from the Department of Critical Care Medicine in Jinhua Municipal Central Hospital for revising the language of the manuscript.

\section{References}

[1] N. Anand and M. H. Kollef, "The alphabet soup of pneumonia: CAP, HAP, HCAP, NHAP, and VAP," Seminars in Respiratory and Critical Care Medicine, vol. 30, no. 1, pp. 3-9, 2009.

[2] C. M. Stoeppel, E. A. Eriksson, K. Hawkins et al., "Applicability of the National Healthcare Safety Network's surveillance definition of ventilator-associated events in the surgical intensive care unit: a 1-year review," Journal of Trauma and Acute Care Surgery, vol. 77, no. 6, pp. 934-937, 2014.

[3] M. Klompas, R. Branson, E. C. Eichenwald et al., "Strategies to prevent ventilator-associated pneumonia in acute care hospitals: 2014 update," Infection Control and Hospital Epidemiology, vol. 35, no. 8, pp. 915-936, 2014.

[4] C. Henriquez-Camacho and J. Losa, "Biomarkers for sepsis," BioMed Research International, vol. 2014, Article ID 547818, 6 pages, 2014.

[5] A. Hasibeder, P. Stein, R. Brandwijk, H. Schild, and M. P. Radsak, "Evaluation and validation of the detection of soluble triggering receptor expressed on myeloid cells 1 by enzymelinked immunosorbent assay," Scientific Reports, vol. 5, Article ID 15381, 2015.

[6] Y. Wu, F. Wang, X. Fan et al., "Accuracy of plasma sTREM-1 for sepsis diagnosis in systemic inflammatory patients: a systematic review and meta-analysis," Critical Care, vol. 16, no. 6, article no. R229, 2012.

[7] S. J. Palazzo, T. A. Simpson, J. M. Simmons, and L. M. Schnapp, "Soluble triggering receptor expressed on myeloid cells-1 (sTREM-1) as a diagnostic marker of ventilator-associated pneumonia," Respiratory Care, vol. 57, no. 12, pp. 2052-2058, 2012.

[8] American Thoracic Society and Infectious Diseases Society of America, "Guidelines for the management of adults with hospital-acquired, ventilator-associated, and healthcareassociated pneumonia," American Journal of Respiratory and Critical Care Medicine, vol. 171, no. 4, pp. 388-416, 2005.

[9] I. A. Du Rand, P. V. Barber, J. Goldring et al., "British Thoracic Society guideline for advanced diagnostic and therapeutic flexible bronchoscopy in adults," Thorax, vol. 66, supplement 3, pp. iiil-iii21, 2011.

[10] F. Michel, B. Franceschini, P. Berger et al., "Early antibiotic treatment for BAL-confirmed ventilator-associated pneumonia: a role for routine endotracheal aspirate cultures," Chest, vol. 127, no. 2, pp. 589-597, 2005.

[11] M. B. Schindler and P. N. Cox, "A simple method of bronchoalveolar lavage," Anaesthesia and Intensive Care, vol. 22, no. 1, pp. 66-68, 1994.

[12] H. Bayram, Ö. Tünger, M. Çivi et al., "Diagnostic and prognostic value of procalcitonin and sTREM-1 levels in sepsis," Turkish Journal of Medical Sciences, vol. 45, no. 3, pp. 578-586, 2015.

[13] N. J. Anand, S. Zuick, J. Klesney-Tait, and M. H. Kollef, "Diagnostic implications of soluble triggering receptor expressed 
on myeloid cells-1 in BAL fluid of patients with pulmonary infiltrates in the ICU," Chest, vol. 135, no. 3, pp. 641-647, 2009.

[14] G. J. Oudhuis, J. Beuving, D. Bergmans et al., "Soluble Triggering Receptor Expressed on Myeloid cells-1 in bronchoalveolar lavage fluid is not predictive for ventilator-associated pneumonia," Intensive Care Medicine, vol. 35, no. 7, pp. 1265-1270, 2009.

[15] C. Palmiere, D. Bardy, P. Mangin, and M. Augsburger, "Value of sTREM-1, procalcitonin and CRP as laboratory parameters for postmortem diagnosis of sepsis," Journal of Infection, vol. 67, no. 6, pp. 545-555, 2013.

[16] G. Horonenko, J. C. Hoyt, R. A. Robbins et al., "Soluble triggering receptor expressed on myeloid cell-1 is increased in patients with ventilator-associated pneumonia: a preliminary report," Chest, vol. 132, no. 1, pp. 58-63, 2007.

[17] F. Duflo, R. Debon, G. Monneret, J. Bienvenu, D. Chassard, and B. Allaouchiche, "Alveolar and serum procalcitonin: diagnostic and prognostic value in ventilator-associated pneumonia," Anesthesiology, vol. 96, no. 1, pp. 74-79, 2002.

[18] S. Gibot and A. Cravoisy, "Soluble form of the triggering receptor expressed on myeloid cells-1 as a marker of microbial infection," Clinical Medicine \& Research, vol. 2, no. 3, pp. 181-187, 2004.

[19] R. M. Determann, J. L. Millo, S. Gibot et al., "Serial changes in soluble triggering receptor expressed on myeloid cells in the lung during development of ventilator-associated pneumonia," Intensive Care Medicine, vol. 31, no. 11, pp. 1495-1500, 2005.

[20] M. Oppert, A. Reinicke, C. Müller, D. Barckow, U. Frei, and K.-U. Eckardt, "Elevations in procalcitonin but not C-reactive protein are associated with pneumonia after cardiopulmonary resuscitation," Resuscitation, vol. 53, no. 2, pp. 167-170, 2002.

[21] S. J. Palazzo, T. Simpson, and L. Schnapp, "Biomarkers for ventilator-associated pneumonia: review of the literature," Heart \& Lung, vol. 40, no. 4, pp. 293-298, 2011.

[22] J.-X. Shi, J.-S. Li, R. Hu et al., "Diagnostic value of sTREM-1 in bronchoalveolar lavage fluid in ICU patients with bacterial lung infections: a bivariate meta-analysis," PLoS ONE, vol. 8, no. 5, Article ID e65436, 2013.

[23] J. R. Delanghe and M. M. Speeckaert, "Translational research and biomarkers in neonatal sepsis," Clinica Chimica Acta, vol. 451, pp. 46-64, 2015.

[24] A. Brzozowska, P. Majak, J. Jerzyñska et al., "Exhaled nitric oxide correlates with IL-2, MCP-1, PDGF-BB and TIMP-2 in exhaled breath condensate of children with refractory asthma," Postepy Dermatologii i Alergologii, vol. 32, no. 2, pp. 107-113, 2015.

[25] M. Navratil, D. Plavec, D. Erceg, S. Bulat Lokas, J. Živković, and M. Turkalj, "Urates in exhaled breath condensate as a biomarker of control in childhood asthma," Journal of Asthma, vol. 52, no. 5, pp. 437-446, 2015.

[26] F. Tahan, H. H. Akar, and B. Saraymen, "Exhaled breath condensate annexin A5 levels in exercise-induced bronchoconstriction in asthma: a preliminary study," Allergologia et Immunopathologia, vol. 43, no. 6, pp. 538-542, 2015. 


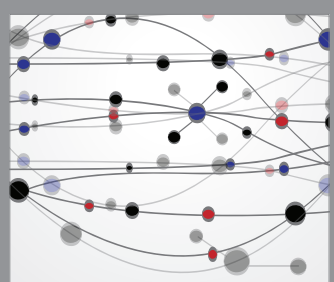

The Scientific World Journal
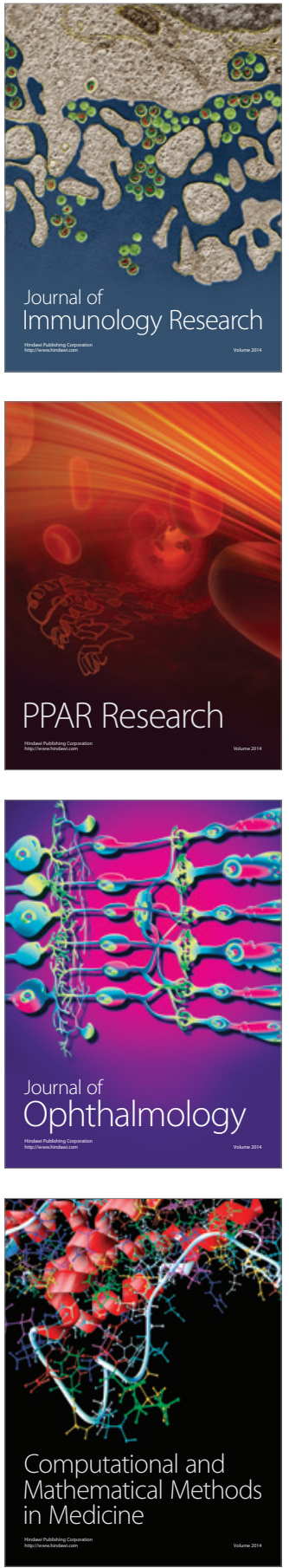

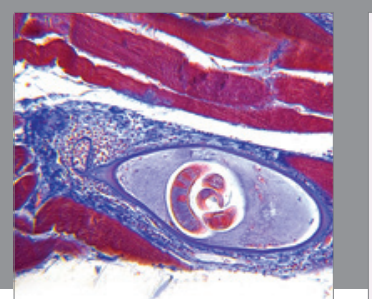

Gastroenterology Research and Practice
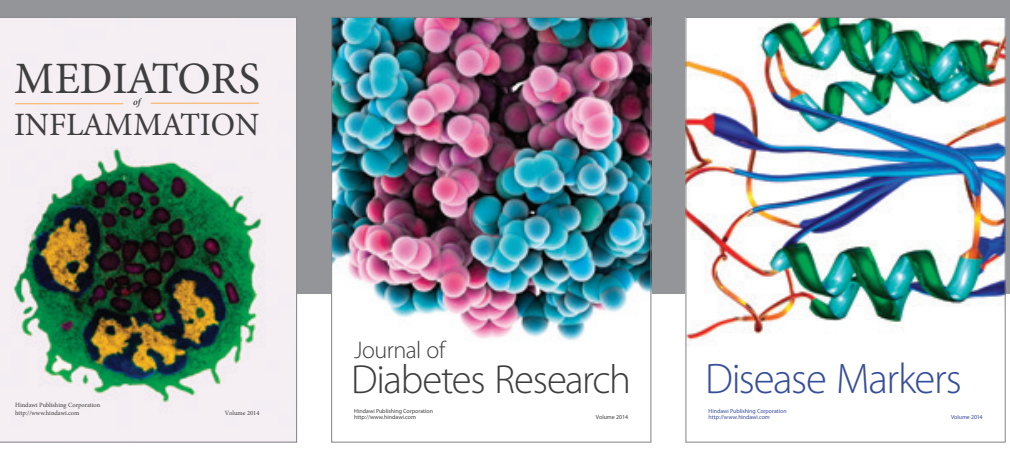

Disease Markers

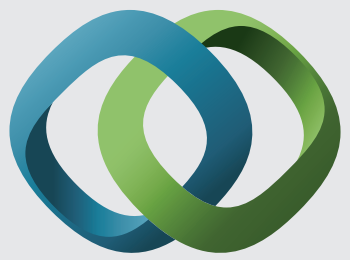

\section{Hindawi}

Submit your manuscripts at

https://www.hindawi.com
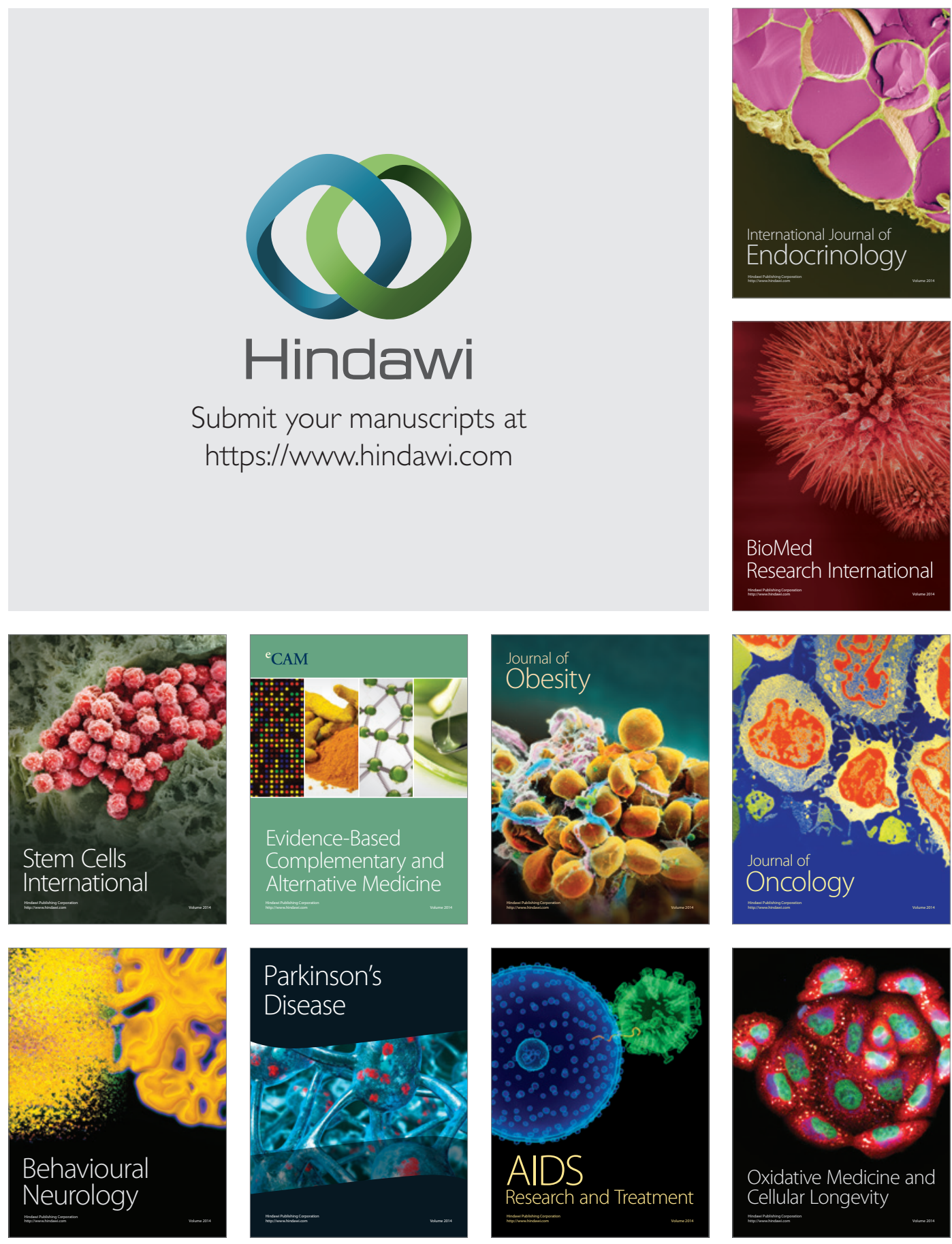